\title{
Morning exercise appears to promote greater fat oxidation and reduce postprandial lipaemic response more than evening exercise
}

\author{
T. Robertson, R. Palmer, A. Doyle, B. Griffin, S. Hampton and A. Collins \\ Division of Nutritional Sciences, Faculty of Health and Medical Sciences, University of Surrey, Guildford, \\ Surrey, GU2 7XH, UK
}

Exercise is known to be beneficial in terms of energy balance ${ }^{(1)}$, body composition and general health but can be difficult to fit into busy schedules. As a result, many of the working population choose to exercise either in the early morning or early evening. However there is little research into the relative physiological and metabolic effects of exercise at different times of day. This study aimed to investigate whether either morning or evening exercise is more beneficial in terms of its effects on respiratory quotient (RQ), energy expenditure (EE), glycaemic and lipaemic responses to a subsequent high fat meal.

Twelve healthy untrained women (mean age $25 \mathrm{y}$ ) were recruited from the university population and randomized to two groups, morning $(n=6)$ and evening $(n=6)$. Each group attended two study days in random order, separated by a minimum of 7 days. The morning group attended at 08:15 h having fasted from 20:00 h the previous evening; the evening group attended at 17:00 h having fasted from 13:00 h. During one visit subjects exercised on a stationary cycle for $22 \mathrm{~min}$ at $60 \% \mathrm{VO}_{2 \max }$ and then consumed a high fat meal (949 kcal, $81 \mathrm{~g}$ fat, $24 \mathrm{~g}$ carbohydrate, $13 \mathrm{~g}$ protein). On the other they consumed the same high fat meal but without prior exercise. On each visit, following the high fat meal, subjects rested supine for $6 \mathrm{~h}$ with half hourly measurement of RQ and EE via indirect calorimetry. Glucose, triacylglycerol (TAG) and non-esterified fatty acids (NEFA) were measured via finger prick blood samples at 15, 30, 60, 90, 120, $180,240,300 \& 360$ min timepoints.

Postprandial RQ for the exercise session was significantly lower than the control session in the morning group $(p \leqslant 0.003)$ but not in the evening group. The magnitude (peak height) of the postprandial TAG response following exercise was significantly lower in the morning than the evening group $(p=0.016)$ (see Figure 1). There was a consistent trend for the TAG area under the curve to be lower following exercise for the morning group $(p=0.076)$ but not for the evening group.

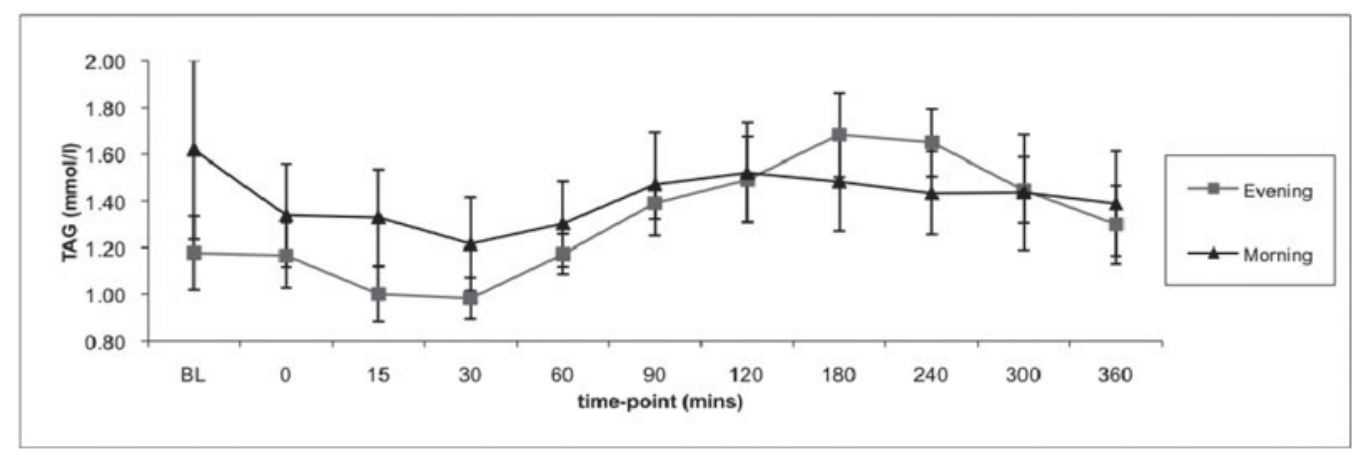

Fig. 1. Plasma postprandial TAG following exercise - comparison between morning and evening groups.

A reduction in RQ following exercise was expected, but only observed in the morning group. Similarly the expected reduction in overall lipaemic response to a high fat meal following exercise was not seen, with only a trend towards a decrease in the morning group and no response in the evening group. This greater response to exercise in the morning may be a result of greater morning insulin sensitivity ${ }^{(2)}$ augmenting lipoprotein lipase (LPL) activity. Increased LPL activity in exercised muscle may explain differences seen as it has been shown to enhance TAG clearance and fatty acid oxidation observed following exercise ${ }^{(3)}$.

These results indicate that morning exercise may have a more beneficial effect in terms of greater fat oxidation (lower RQ) and a reduced postprandial lipaemia than evening exercise in untrained individuals.

1. Poehlman ET, Melby CL, Badylak SF \& Calles J (1989) Metabolism 38, 85-90.

2. Morgan LM, Aspostolakou F, Wright J \& Gama R (1999) Ann Clin Biochem 36, 447-50.

3. Herd SL, Kiens B, Boobis LH \& Hardman AE (2001) Metabolism 50, 756-762. 